\title{
Next Generation Life Support Project: Development of Advanced Technologies for Human Exploration Missions
}

\author{
Daniel J. Barta, Ph.D. ${ }^{1}$ \\ NASA Johnson Space Center, Houston, Texas, 77058
}

\begin{abstract}
Next Generation Life Support (NGLS) is one of several technology development projects sponsored by the National Aeronautics and Space Administration's Game Changing Development Program. NGLS is developing life support technologies (including water recovery, and space suit life support technologies) needed for humans to live and work productively in space. NGLS has three project tasks: Variable Oxygen Regulator (VOR), Rapid Cycle Amine (RCA) swing bed, and Alternative Water Processing. The selected technologies within each of these areas are focused on increasing affordability, reliability, and vehicle self sufficiency while decreasing mass and enabling long duration exploration. The RCA and VOR tasks are directed at key technology needs for the Portable Life Support System (PLSS) for an Exploration Extravehicular Mobility Unit (EMU), with focus on prototyping and integrated testing. The focus of the Rapid Cycle Amine (RCA) swing-bed ventilation task is to provide integrated carbon dioxide removal and humidity control that can be regenerated in real time during an EVA. The Variable Oxygen Regulator technology will significantly increase the number of pressure settings available to the space suit. Current spacesuit pressure regulators are limited to only two settings while the adjustability of the advanced regulator will be nearly continuous. The Alternative Water Processor efforts will result in the development of a system capable of recycling wastewater from sources expected in future exploration missions, including hygiene and laundry water, based on natural biological processes and membrane-based post treatment. The technologies will support a capability-driven architecture for extending human presence beyond low Earth orbit to potential destinations such as the Moon, near Earth asteroids and Mars.
\end{abstract}

\section{Nomenclature}

$\begin{array}{ll}\mathrm{CO}_{2} & =\text { carbon dioxide } \\ \mathrm{ft}^{2} & =\text { square foot } \\ \mathrm{kg} & =\text { kilograms } \\ \mathrm{kW} & =\text { kilowatts } \\ \mathrm{lb} & =\text { pounds } \\ \mathrm{LiOH} & =\text { lithium hydroxide } \\ \mathrm{mg} & =\text { milligrams } \\ \mathrm{NH}_{4}{ }^{+} & =\text {ammonium } \\ \mathrm{NO}_{x}^{-} & =\text {nitrates and nitrites } \\ \mathrm{O}_{2} & =\text { oxygen } \\ \mathrm{sec} & =\text { seconds }\end{array}$

\section{Introduction}

$\mathrm{T}$ he President's Fiscal Year (FY) 2012 budget request ${ }^{1}$, consistent with the 2011 NASA Strategic Plan ${ }^{2}$, outlined an approach to strengthen the United States' human space flight activities by transitioning from an engineering focus on building the ISS to an emphasis on scientific research and technology development - essential building blocks for a long-term human space exploration program. The ISS will be a centerpiece for NASA's planning for extended space missions, as it will serve as a research laboratory and technology test bed. Technology investments

\footnotetext{
${ }^{1}$ Project Manager, 2101 NASA Parkway, Houston, TX 77058, Mail Code: EC6, AIAA Senior Member.
} 
are expected to lay the foundation for travel beyond low-Earth-orbit, including destinations such as cislunar space and Lagrangian points, the Moon, asteroids, the moons of Mars and Mars itself ${ }^{3}$. In the budget, NASA's Space Technology initiative was given additional emphasis by separating it from Aeronautics Research and incorporating technology development from NASA's Exploration Systems Mission Directorate (ESMD). Space Technology is directed to develop critical space technologies through multi-phased technology development efforts, demonstrations, competitive opportunities, and partnerships, engaging Government, industry, and academia. The Office of the Chief Technologist (OCT) manages Space Technology.

NASA's Space Technology Program will perform "push" technology development and demonstration, to enable new approaches to achieving NASA's current mission set and future missions not feasible today. This approach is in contrast to the mission-focused technology development activities within the NASA Mission Directorates, which "pull" technology development based upon established Mission needs. Space Technology has three main technology divisions: Early Stage Innovation, Game Changing Technology and Cross-Cutting Capability Demonstration, spanning all phases of technology development from basic research to proof of concept, technology maturation to flight demonstration and infusion. During Fiscal Year (FY) 2011, technology development for Extravehicular Activity (EVA) and Environmental Control and Life Support (ECLSS), were performed under separate Foundational Domains within ESMD's Exploration Technology Development and Demonstration (ETDD) Program. Beginning in FY 2012, EVA Portable Life Support System (PLSS) and ECLSS technology development were moved to Space Technology under the Next Generation Life Support (NGLS) Project. NGLS has a much leaner and narrower portfolio than its predecessors, Life Support and Habitation Systems (LSHS) ${ }^{4}$ and EVA Technology. This paper will provide an overview of the NGLS Project, including its organization and technical content.

\section{Next Generation Life Support (NGLS) Project}

NGLS is one of about 20 active technology development projects under Space Technology's Game Changing Development Program (GCDP). GCDP seeks to identify and rapidly mature innovative, high-impact capabilities and technologies and complement them with "new start" and competitively-selected projects by using a balanced approach of guided technology development efforts and competitively-selected efforts from across NASA, academia, industry and other government agencies. The Program has seven theme areas: Safe and Reliable Human Exploration (NGLS is within this theme); Intelligent Space Operations; Advanced Materials, Structures, and Manufacturing; Affordable Space Exploration; Revolutionary Robotic Systems; Advanced Measurement, Navigation and Communication Systems; and Future Energy Systems. Projects must address Technical Area key technical challenges within the Space Technology Roadmap ${ }^{5}$ and be relevant to one or more Space Technology Grand Challenges 6 .

The NASA Space Technology Program Resource Guidance (PRG) FY 2013 directed the Next Generation Life Support (NGLS) Project to develop life support technologies (including atmospheric revitalization, water recovery, and space suit life support technologies) needed for humans to live and work productively in space. The primary project goal is to advance technologies Technology Readiness Level (TRL) and infuse the technologies into Advanced Exploration Systems (AES) system demonstrations. Within each of the prescribed focus areas, the most challenging (or high payoff) technology solutions were selected to be included as part of this project. The selected technologies will provide new capabilities not found in existing NASA systems. The selected technologies within each of these areas are focused on increasing affordability, reliability, performance and vehicle self sufficiency while decreasing mass and enabling long duration exploration.

The NGLS project originally planned to carry out five technology development tasks including: Suit Water Membrane Evaporator (SWME), Rapid Cycle Amine (RCA) swing bed, Variable Oxygen Regulator (VOR), Advanced Oxygen Recovery and Alternative Water Processor, with work initiating in all technical

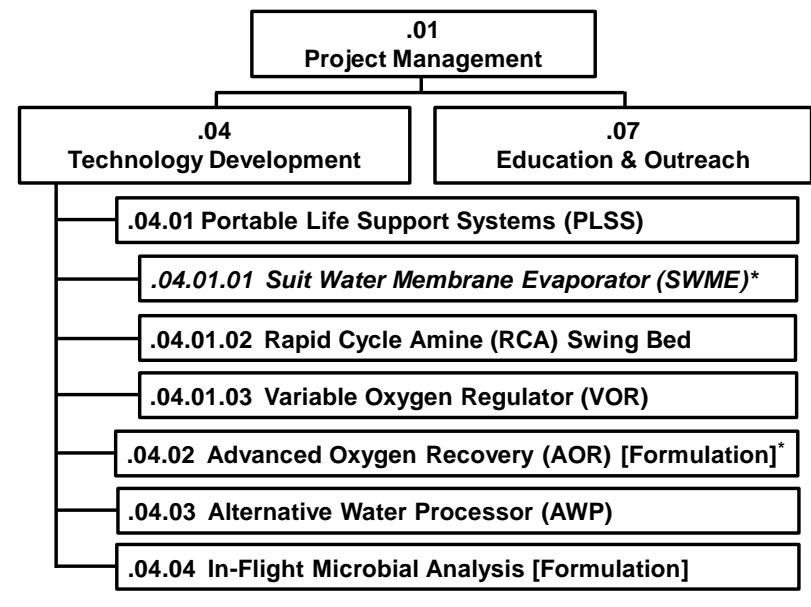

Figure 1. Work Breakdown Structure.

*SWME and AOR started as full development tasks but were descoped early in the project when budgets were reduced. 
areas at the beginning of the FY 2012. Following approval of the Appropriation to NASA mid-November, 2011, NGLS content was adjusted to include only three technology development tasks, RCA, VOR, and Alternative Water Processor, with two formulation tasks, Advanced Oxygen Recovery and In-Flight Microbial Monitoring. The Work Breakdown Structure (WBS) on which project organization is based is shown in Figure 1. The project plan and WBS follow NASA procedural and management requirements technology program and project management. ${ }^{7}$ Only the sections that are used are included in the WBS. Technical work is performed across four NASA field centers: Johnson Space Center (JSC), Ames Research Center (ARC), Marshall Space Flight Center (MSFC) and Kennedy Space Center (KSC), with involvement of several outside institutions including Texas Tech University, University of Puerto Rico, Carleton Technologies and Hamilton Sundstrand.

Table 1 lists Key Performance Parameters (KPP) that the technologies developed under this project will strive to meet. State of the Art (SOA) values for these KPPs are listed for comparison. The Research and Technology Development (R\&TD) threshold values and goals illustrate the advances that this project strives to make. KPPs will be evaluated either through analysis or testing.

Figure 2 provides a top-level schedule for the project with major milestones identified. NGLS is funded for two years with an optional third year. Continuation reviews will be held at the end of each year to gauge progress and approve the next year's funding. The third year will be competitive and is not guaranteed.

The following sections describe in more detail each of the three main technology tasks within NGLS, the RCA, VOR and AWP. The tasks that were de-scoped from the project or are in formulation, will not covered within this paper.

\section{A. Portable Life Support System (PLSS) Tasks}

The RCA and VOR tasks are directed at key technology needs for the next-genertation Portable Life Support System (PLSS) for an Exploration Extravehicular Mobility Unit (EMU), with focus on prototyping and integrated testing. The focus of the RCA development is to provide an integrated $\mathrm{CO}_{2}$ removal and humidity control system that can be regenerated in real time during an $\mathrm{EVA}^{8}$. Not only does this capability eliminate consumables associated with non-regenerable technologies (e.g. $\mathrm{LiOH}$ ), the RCA eliminates off-suit regeneration that requires ancillary equipment and power (Metal Oxide), and eliminates $\mathrm{CO}_{2}$ scrubbing as an EVA duration limitation ${ }^{9,10}$. The project is also targeting a mass reduction of $67 \%$ as compared to the state of the art (Metal Oxide). The objective of the VOR technology development activity is to significantly increase the number of pressure settings on the pressure regulator. Current spacesuit pressure regulators are limited to only two settings. The VOR design will incorporate a stepper motor yielding multiple settings (to exceed 4,000 over the pressure range) ${ }^{11}$. The enhanced performance would facilitate and improve EVA operations and pre-breathe protocols, allow regulation of suit pressure to match different vehicle pressures including integration with suit ports, allow for in-suit decompression sickness (DCS) treatment, and minimize or eliminate prebreathe durations prior to an EVA ${ }^{9,11}$. The PLSS technologies will be

Table 1. Key Performance Parameters

\begin{tabular}{|c|c|c|c|}
\hline Technical Area & $\begin{array}{l}\text { State of the } \\
\text { Art (SOA) }\end{array}$ & $\begin{array}{c}\text { Threshold } \\
\text { Values }\end{array}$ & R\&TD Goals \\
\hline \multicolumn{4}{|c|}{ Rapid Cycle Amine (RCA) Swing Bed } \\
\hline $\mathrm{CO}_{2}$ removal system mass $(\mathrm{kg})$ & $60.8^{*}$ & $\leq 15.5$ & 5 \\
\hline System Life (EVA uses) & 25 & $\geq 50$ & $100+$ \\
\hline $\mathrm{CO}_{2}$ removal rate $(\mathrm{kg} / \mathrm{sec})$ & 2.26 & 2.26 & 3.04 \\
\hline \multicolumn{4}{|c|}{ Variable Oxygen Regulator (VOR) } \\
\hline Pressure Settings & 2 & $\geq 5$ & 84 \\
\hline Pressure Range & $\sim 0.9 \& 4.3$ & $0.3-8.4$ & $0-8.4$ \\
\hline Contamination Tolerance & $<2 \mathrm{mg} / \mathrm{ft}^{2}$ & $\geq 2 \mathrm{mg} / \mathrm{ft}^{2}$ & $50 \mathrm{mg} / \mathrm{ft}^{2}$ \\
\hline Mass (lb) & $\mathrm{NA}^{*}$ & $\leq 8$ & 3.5 \\
\hline \multicolumn{4}{|c|}{ Alternative Water Processor (AWP) } \\
\hline Wastewater Recycling $^{\dagger}$ & $0 \%$ & $\geq 85 \%$ & $>95 \%$ \\
\hline Consumable Reduction from SOA & - & $\leq 20 \%$ & $50 \%$ \\
\hline
\end{tabular}




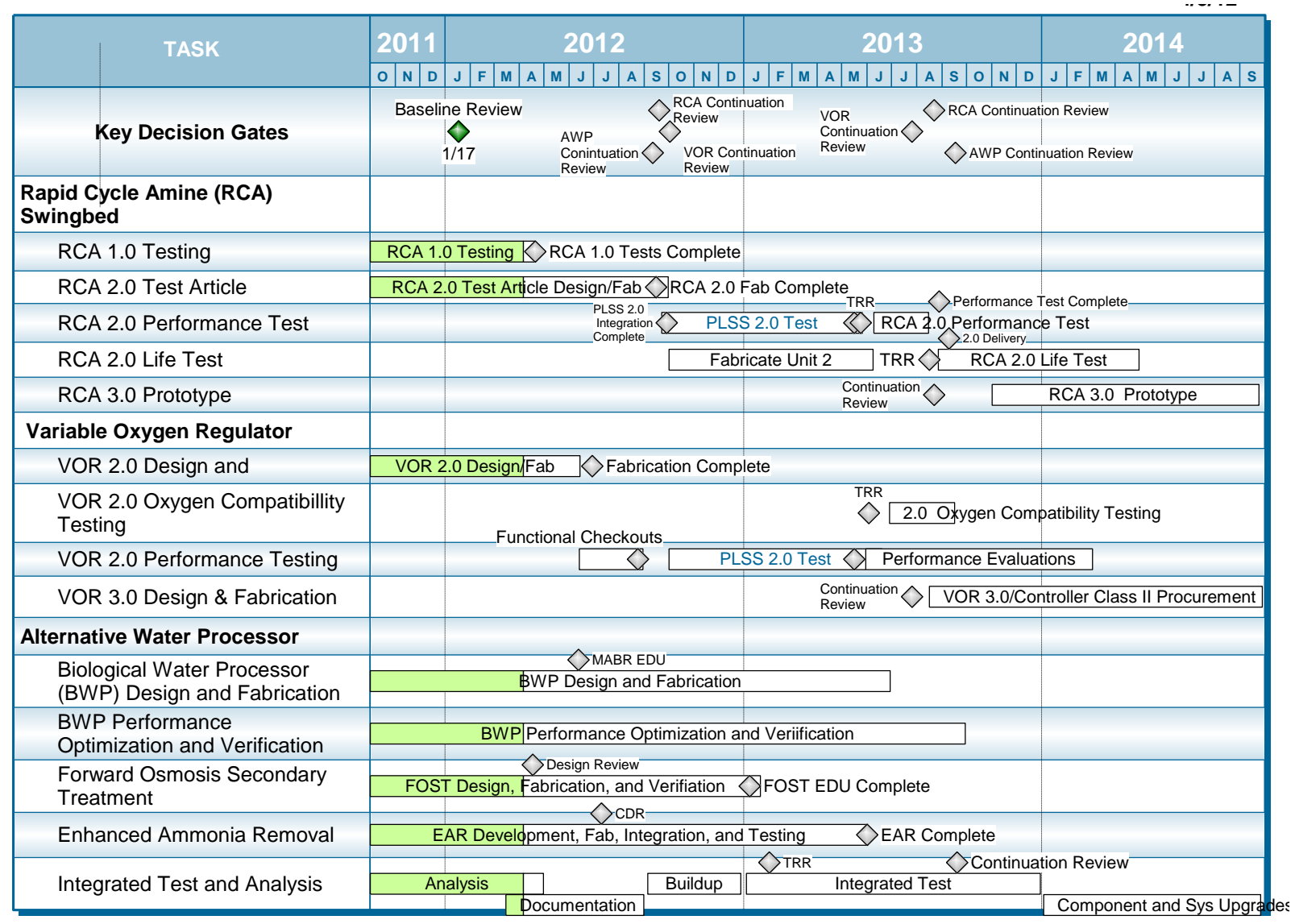

Figure 2. Top Level Schedule with Major Milestones

evaluated in integrated tests performed in cooperation with the Advanced Exploration Systems (AES) Advanced EVA Systems Development Project. Funding during the first two years would lead to TRL 5 hardware tested in the PLSS 2.0 integrated tests. The third year would include development of prototype hardware for inclusion in manned EVA integrated testing, ultimately leading to TRL 6.

\section{Rapid Cycle Amine (RCA) Swing Bed}

The RCA is under development to provide regenerable $\mathrm{CO}_{2}$ and humidity control for the EVA crew member, not available with the Shuttle era Extra-vehicular Mobility Unit (EMU) ${ }^{9,10}$. It provides a capability for real-time, onback regeneration of the sorbent. The current EMU uses either a lithium hydroxide ( $\mathrm{LiOH})$ canister or a Metal Oxide (Metox) canister. When $\mathrm{LiOH}$ is used, it cannot be regenerated and becomes a mission consumable. Metox canisters must be regenerated using a special oven onboard ISS. This requires some crew time and significant power (over $1 \mathrm{~kW}$ for as long as 14 hours). Both of these state-of-the-art (SOA) technologies occasionally "break through," an in this event the EVA must be terminated prematurely. The RCA therefore reduces consumables, logistics, crew time, and eliminates $\mathrm{CO}_{2}$ removal as an EVA duration limiting consumable. The amine used in the swing bed also removes water vapor from the suit ventilation loop eliminating the need for a condensing heat exchanger, slurper, and rotary separator.

The RCA design consists of alternating absorbing and desorbing beds to contain amine coated pellets mounted in an aluminum foam substrate ${ }^{8}$. A valve is required to cycle the beds between the ventilation flow and vacuum exposure. Early proof-of-concept development of the RCA investigated rectangular and cylindrical canisters ${ }^{12}$, and three experimental valve types to cycle between beds, a spool valve, ganged ball valve, and drum valve. A trade study, heavily based on test data and a correlated math model, suggested the rectangular canister be pursued for future development work. RCA version 1.0 is pictured in Figure 3.

This project will mature the technology from proof-of-concept by building a full sized assembly, RCA 2.0, which will consist of an integrated rectangular canister and ganged ball valve. The ball valve concept was selected for this iteration based on the valve reliability from related projects. RCA 2.0 will be fabricated, tested, and 
evaluated in cooperation with the AES Advanced EVA Systems Development project in their packaged PLSS (PLSS 2.0) integrated test, which will involve vacuum chamber testing of a packaged PLSS using a metabolic simulator and nitrogen gas as the working fluid. Should development be continued into a third year, a high fidelity oxygen compatible unit will be developed (RCA 3.0) for evaluation in human vacuum chamber tests with $100 \%$ oxygen as the working fluid.

\section{Variable Oxygen Regulator (VOR)}

The VOR will provide variable suit pressure settings between 0 and 8.4 psid above the ambient environment pressure. The current EMU only has two settings that are mechanically controlled through an intricate mechanical linkage. This new capability will

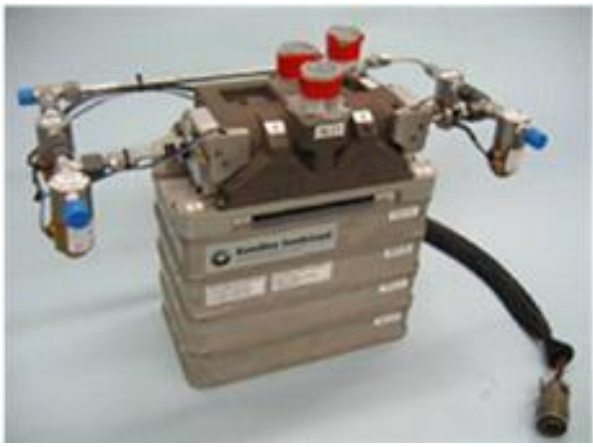

Figure 3. Rapid Cycle Amine Swing Bed Unit 1.0 enable suitport operations, in-suit decompression sickness treatment, flexibility for interfacing to different vehicles and the ability to run variable pressure profiles during an EVA. A variable pressure profile could consist of starting the EVA at a higher pressure, ramping down to a lower pressure at a controlled rate, and then increasing the pressure again prior to returning to the vehicle. This ability gives advantages that include decreasing prebreathe time, maximizing mobility and therefore minimizing crew fatigue, and also minimizing gas loss due to suit leakage, ${ }^{9,11}$.

The current VOR concept consists of a dual stage regulator in which the second stage uses a stepper motor to control the amount of compression on a spring that is balanced against the gas pressure and then controls the size of the orifice that feeds gas into the suit ${ }^{11}$. Previous efforts included trading this electromechanical control method against a design that relied on a pressure feedback control scheme. An initial regulator was built and tested, using nitrogen as the working fluid, across a wide range of inlet pressures and demand flow rates (Figure 4).

Two second generation units, VOR 2.0, will be designed with an integrated controller for use with high pressure oxygen and will be delivered during the project's first year. These test articles will be subjected to a range of performance testing which will include development of a control algorithm to drive the stepper motor. The regulators will be integrated into the PLSS 2.0 architecture as PLSS primary and secondary oxygen regulators, in collaboration with the AES Advanced EVA Systems Development Project for integrated testing. The regulators will also be evaluated for oxygen compatibility. Findings will be used to mature the technology and design high fidelity units (VOR 3.0), which will be built, if third year of funding is approved, to support future human in-theloop testing.

\section{Alternative Water Processor (AWP)}

The Alternative Water Processor (AWP) efforts will result in the development of a system capable of recycling wastewater from sources expected in future exploration missions, including hygiene and laundry water, using a "disruptive" technology based on natural biological processes. The AWP is being designed to recycle more than 95\% of exploration wastewater, increasing closure compared to the state of the art. The AWP could ultimately replace the funcsions of the Urine Processor Assembly (UPA) and reduce or eliminate the need for the multifiltration beds of the Water Processor Assembly (WPA) of the International Space Station (ISS) ${ }^{13}$.

At the center of the AWP are two unique game changing technologies: 1) a biological water processor (BWP) to mineralize organic forms of carbon and nitrogen and 2) an advanced membrane processor (Forward Osmosis Secondary Treatment) for removal of solids and inorganic ions. In addition, "greener" and less toxic methods for wastewater stabilization will need to be utilized. Biological processing is expected require fewer resources while providing necessary system performance: $>90 \%$ removal of wastewater organics including surfactants, $\mathrm{pH}$ stabilization, oxidation of $\mathrm{NH}_{4}{ }^{+}$to $\mathrm{NO}_{\mathrm{x}}{ }^{-}$(up to $80 \%$ ), and improved properties of concentrated brines ${ }^{14},{ }^{15}$. The Forward Osmosis Secondary Treatment (FOST) system is a membrane processor that uses both forward osmosis (FO) and reverse osmosis (RO) and can easily tolerate wastewaters high in non-volatile organic content and solids associated with shower and/or hand washing. These contaminants have good rejection performance but high membrane fouling potential using conventional pressure-driven membrane techniques. The FOST approach addresses this issue through incorporation of FO pretreatment which allows RO to be used to reject these

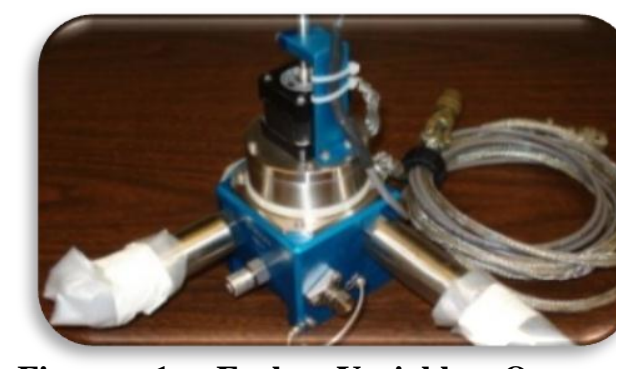

Figure 1. Early Variable Oxygen Regulator bench-top prototype. 
contaminants effectively without premature fouling due to micellar surfactant aggregates. The FOST is a off-shoot a prior water processor technology, Direct Osmotic Concentration ${ }^{16}$. A top level schematic of the AWP is shown in Figure 5. The first year involves development of unit technologies, including a Biological Water Processor (BWP) and Forward Osmosis Secondary Treatment (FOST) system. The technologies will be integrated and evaluated as a system during the second year of the project. The third year, if funded, will include extended systems testing, upgrades to unit technologies and a final integrated test bringing the system to TRL 4-5. The AWP will be delivered to the AES Water Recovery Project for technology infusion when work is completed.

\section{Biological Water Processor (BWP)}

During the first year of effort, several BWP concepts will be considered. In cooperation with Texas Tech University, a singlestage Membrane-Aerated Biological Reactor (MABR) ${ }^{14,15}$ will be investigated as the primary option. Alternative options include fixed-shell two-stage reactor designs from Kennedy Space Center (KSC) which separate oxidation of organic carbon from nitrification. Johnson Space Center (JSC) will evaluate urine pretreatment alternatives with lower toxicity levels and having compatibility with biological water processors. Concomitantly, an equivalent systems mass (ESM) evaluation and system architecture study will be performed to evaluate single stage versus two stage reactor designs.

\section{Forward Osmosis Secondary Treatment (FOST)}

The Forward Osmosis Secondary Treatment (FOST) subsystem will be designed to treat the effluent of the primary treatment biological system. The FOST subsystem will be designed to remove suspended and dissolved solids including mineral salts in the processed wastewater effluent from the bioreactor. A supporting subsystem within the FOST will be developed in cooperation with the University of Puerto Rico, termed Electrochemical Ammonia Removal (EAR). The purpose of the EAR is to remove any un-oxidized nitrogen that may be present in the bioreactor effluent, which should improve the quality of the FOST product water. ARC will complete design of the FOST during the first year of effort, and complete fabrication early in the second year. The FOST system will be tested to verify it meets design specifications before being delivered to Johnson Space Center (JSC) for integration as part of the Alternative Water Processor.

\section{Systems Analysis, Integration and Testing}

Systems analysis will be used to perform trade studies supporting gate reviews, support development of design specifications, analyze test data, and model the selected processes.

The design of the integrated system architecture will be baselined early in the second-year. The selected bioreactor approach will be integrated with the FOST system. An integrated system test will be performed. The EAR will be integrated after the integrated test is underway. Microbiological analysis will be performed on the integrated system and investigations on reactor start-up (microbial inoculation) and reactor resiliency (community stability) will be carried out. During the optional third year the integrated test would be continued. Unit processors and systems design will be upgraded based on lessons learned and a new integrated systems level test would be performed.

\section{Summary}

The NGLS Project is one of several Exploration Technology Development projects within Space Technology's Game Changing Development Program (GCDP). GCDP seeks to identify and rapidly mature innovative/high impact capabilities and technologies that may lead to entirely new approaches for the Agency's future space 
missions. The three main technology tasks under NGLS, the Rapid Cycle Amine Swing Bed, Variable Oxygen Regulator and Alternative Water Processor have the potential to revolutionize life support systems in EVA suits and human exploration spacecraft of the future, by removing limitations of the current SOA, by adding new capability and/or utilizing unconventional approaches, while improving performance over the state of the art.

\section{Acknowledgments}

The author wishes to thank Chantel Whatley and NGLS Task Leads, including Karen Pickering (Advanced Water Processor), Neil Townsend (All EVA tasks), Colin Campbell (VOR and RCA), Michael Flynn (FOST), as well as other members of the NGLS Project Team.

\section{References}

${ }^{1}$ Bolden, C. F., "NASA FY 2012 Budget Request Executive Summary," National Aeronautics and Space Administration, Washington, DC, February 2011.

${ }^{2}$ Office of the Chief Financial Officer, "2011 NASA Strategic Plan," National Aeronautics and Space Administration, Washington, DC, NP-2011-01-699-HQ, February 14, 2011.

${ }^{3}$ Augustine, N. R., "Seeking a Human Spaceflight Program Worthy of a Great Nation," Review of U.S. Human Spaceflight Plans Committee, Washington, DC, October 2009.

${ }^{4}$ Barta, D. J., and McQuillan, J., "Life Support and Habitation Systems: Crew Support and Protection for Human Exploration Missions Beyond Low- Earth Orbit," AIAA-2011-5095, 41 ${ }^{\text {st }}$ International Conference on Environmental Systems; 17-21 July 2011; Portland, Oregon; United States.

${ }^{5}$ Colladay, R. S., "NASA Space Technology Roadmaps and Priorities: Restoring NASA's Technological Edge and Paving the Way for a New Era in Space", Steering Committee for NASA Technology Roadmaps; National Research Council of the National Academies, PDF Book \#13354, ISBN 978-0-309-25362-8, The National Academies Press, Washington, D.C., 2012.

${ }^{6}$ Strategic Integration Office, "Space Technology Grand Challenges", Office of the Chief Technologist, National Aeronautics and Space Administration, Washington, D.C., 2010.

${ }^{7}$ Office of the Chief Engineer, "NASA Research and Technology Program and Project Management Requirements," National Aeronautics and Space Administration, Washington, DC, NPR 7120.8, November 24, 2010.

${ }^{8}$ Papale, W., Paul, H., and Thomas, G., "Development of Pressure Swing Adsorption Technology for Spacesuit Carbon Dioxide and Humidity Removal", 36th International Conference on Environmental Systems, SAE Paper Number 2006-01-2203, 2006.

${ }^{9}$ Chullen, C., and Westheimer, D. T., "Extravehicular Activity Technology Development Status and Forecast," AIAA-2011-5179, 41 ${ }^{\text {st }}$ International Conference on Environmental Systems; 17-21 July 2011; Portland, Oregon; United States.

${ }^{10}$ Swickrath, M. J., Anderson, M., McMillin, S., and Broerman, C., "Simulation and Analysis of Vacuum Swing Adsorption Units for Spacesuit Carbon Dioxide and Humidity Control," AIAA-2011-5243, 41 ${ }^{\text {st }}$ International Conference on Environmental Systems; 17-21 July 2011; Portland, Oregon; United States.

${ }^{11}$ Mosher, M., and Campbell, C., "Design and Testing of a Variable Pressure Regulator for a Flexible Spacesuit Architecture," 40th International Conference on Environmental Systems, AIAA 2010-6064, Barcelona, Spain, July $11-15,2010$.

${ }^{12}$ Paul, H., and Rivera, F. L., "Spacesuit Portable Life Support System Rapid Cycle Amine Repacking and Subscale Test Results," 40th International Conference on Environmental Systems, AIAA 2010-6066, Barcelona, Spain, July 11-15, 2010.

${ }^{13}$ Carter, L., "Status of the Regenerative ECLS Water Recovery System," 40th International Conference on Environmental Systems, AIAA 2010-6216, Barcelona, Spain, July 11-15, 2010.

${ }^{14}$ Jackson, W. A., Peterson, K., Morse, A. and Landes, N., "Development and Testing of a TRL 5 Bioreactor for Pretreatment of a Lunar Surface Wastestream," 40th International Conference on Environmental Systems, AIAA 2010-6239, Barcelona, Spain, July 11-15, 2010.

${ }^{15}$ Jackson, W. A., Christenson, D., Kubista, K., Morse, A., Morse, S., Vercellino, T., and Wilson, D., "Performance of a TRL 5 Bioreactor for Pretreatment of an Extended Habitation Wastestream," AIAA-2011-5132, $41^{\text {st }}$ International Conference on Environmental Systems; 17-21 July 2011; Portland, Oregon; United States. 